2. Lee, J.H. et al. Ann. Clin. Transl. Neurol. 1, 2-4 (2014).

3. McEwen, A.E. Mol. Biol. Cel/ 25, 2365-2374 (2014)

4. Zanin-Zhorov, A. et al. Proc. Natl. Acad. Sci. USA 111 16814-16819 (2014).

5. Flynn, R. et al. Blood 127, 2144-2154 (2016)

6. Weiss, J. et al. ROCK2 controls Th17 cytokine-driven induction of $T$ follicular helper cells via competitive antagonism of STAT3 and STAT5 activation. Science Signaling, in the press.

7. Rigen, M. et al. Cancer Res. 74.19 (Suppl.), 1827 (2014).

8. Tonra, J.R. et al. Cancer Res. 75.15 (Suppl.), 2590 (2015).

9. Jhaveri, K.L. et al. J. Clin. Oncol. 33 (Suppl.), 609 (2015)

10. Zanin-Zhorov, A. et al. J. Immunol. 192 (Suppl.), 63.3 (2014) http://www.jimmunol. org/content/192/1_Supplement/63.3?related urls=yes\&legid=jimmunol;192/1 Supplement/63.3.

11. Zanin-Zhorov, A., et al. J. Immunol. 194 (Suppl.), 140.15 (2015). http://www.jimmunol.org/content/194/1 Supplement/140.15.abstract? sid=c732cfab-30a64050-b7ab-cdc4033c01c4.

12. Weiss, J. et al. J. Immunol. 194 (Suppl.), 121.7 (2015). http://www.jimmunol.org/content/194/1_Sup plement/121.7.abstract?sid=c732cfab-30a6-4050b7ab-cdc4033c01c4.

13. Zanin-Zhorov, A. et al. Am. Soc. Hematol. abstr. 540 (2014). https://ash.confex.com/ash/2014/webprogram/
Paper73112.html.

14. Zanin-Zhorov, A. et al. FOCiS 2013 abstr. OR.11 (2013). http://www.focisnet.org/images/events/focis2013/ Abstract\%20Supplement FINAL 06272013.pdf. 15. Weiss, J. et al. FOCiS 2015 abstr. OR.08 (2015).

\section{Nature Biotechnology replies:}

We welcome Kadmon's statement that it "has a responsibility to share its discoveries with the scientific community in peerreviewed journals." Nature Biotechnology did contact Kadmon to inquire about its publication policies when preparing the Editorial $^{1}$; at that time, the company did not respond to questions about its publication policy. The date of October 2014 was chosen because no papers were shown on Kadmon's website (http://kadmon.com/news/ publications/) earlier than that date, which is five years after the company was founded.

1. Anonymous. Nat. Biotechnol. 34, 115 (2016).

\title{
Secure cloud computing for genomic data
}

\section{To the Editor:}

The 'cloud' offers scalable and competitively priced computing resources for the analysis and storage of data from large-scale genomics studies, but it must also ensure that human subject data are hosted in a context that is both secure and compliant with regulations. Concerns continue to surface in recent years regarding potential hacking and potential loss of patient privacy of data held within the cloud ${ }^{1-3}$. Security challenges for human genomics research in the cloud include researcher's and administrator's lack of familiarity with security features and inconsistent guidelines from different regulatory bodies for genomic datasets. These inconsistencies can cause confusion as to whether or not security requirements are being met on cloud for a particular human dataset. Ultimately, if a research environment in the cloud is not compliant with regulatory guidelines, this could mean that an investigator might lose access to a dataset. Here, we lay out a set of cloud security guidelines that meet a wide range of regulatory requirements. Our intent is twofold: first, to raise awareness regarding the variability and complexity of current guidelines; and second, to highlight how redundant security assessments that are currently carried out dataset by dataset in the cloud could be replaced by a 'do-once, use-many-times' framework. We envision that the do-once, use-many-times guidelines provided here will help researchers navigate various cloud solution providers and will ultimately yield savings in costs, time and staffing. Although the cloud technology stack will continue to evolve rapidly, which in turn will change the specifics of solution implementation, we believe that the security requirements presented here will be applicable for the foreseeable future.

At Stanford University (Stanford, CA), we have developed a secure Google Cloud gateway to support multiple research projects, including datasets at the US National Institutes of Health (NIH; Bethesda, $\mathrm{MD}$ ), other government agencies and private institutions. In research, human genomic sequence data are de-identified via Safe Harbor ${ }^{4}$ guidelines of personally identifiable information (PII; https://en.wikipedia.org/ wiki/Personally_identifiable_information), and associated health data are stripped of protected health information (PHI; https:// en.wikipedia.org/wiki/Protected_health information). The resulting de-identified data can be divided into three categories: first, public data; second, data not classified as $\mathrm{PHI}$ or PII; and third, data very rarely classified as PHI or PII. Typically, public data do not need to meet any security requirements. And PHI or PII data held by a covered entity (see https://privacyruleandresearch.nih. gov/pr_06.asp) need to comply with Health Insurance Portability and Accountability Act (HIPAA; http://www.hhs.gov/hipaa/index. html) rules. However, data that are not PHI or PII are subject to a nonstandard set of regulations from various regulatory bodies. These regulatory bodies can be federal government agencies, such as the NIH, or private institutions like a hospital or business or an Institutional Review Board (IRB).

To illustrate just how inconsistent current guidelines are-for non-PHI data-one need only look at the frequent requirement of IRBs that cloud providers show an ability to comply with HIPAA rules. IRBs do this not because the data require compliance with HIPAA rules but rather because HIPAA compliance serves as a proxy for satisfactory cloud security features. Another concern of IRBs is that sometime in the future ${ }^{5}$, de-identified whole-genome data may be classified as PHI more frequently

\begin{tabular}{|c|c|c|c|c|}
\hline & Infrastructure security & \multicolumn{2}{|c|}{ Data access controls } & Security management \\
\hline \multirow{2}{*}{$\begin{array}{l}\text { Institutional } \\
\text { administrator }\end{array}$} & $\begin{array}{l}\text { Implement } \\
\text { institutional firewalls }\end{array}$ & $\begin{array}{l}\text { Integrate with } \\
\text { institutional } \\
\text { authentication }\end{array}$ & $\begin{array}{l}\text { Implement 'Principle } \\
\text { of Least Privilege' }\end{array}$ & $\begin{array}{l}\text { Set up monitoring } \\
\text { and reactive breach } \\
\text { management }\end{array}$ \\
\hline & $\begin{array}{l}\text { Review logical and } \\
\text { physical security }\end{array}$ & $\begin{array}{l}\text { Establish data } \\
\text { privacy } \\
\text { agreement }\end{array}$ & $\begin{array}{l}\text { Enable } \\
\text { encryption }\end{array}$ & Turn on logging \\
\hline \multirow{2}{*}{$\begin{array}{l}\text { Cloud } \\
\text { provider }\end{array}$} & Logical security & \multicolumn{2}{|c|}{ Encryption } & \\
\hline & Physical security & Authentication & Authorization & Logging \\
\hline
\end{tabular}

Figure 1 Security stack. High-level features-infrastructure security, data access controls and security management (dark blue boxes) - that cloud security must meet for genomics research. The boxes in the bottom two rows present the cloud infrastructure-as-a-service (laaS) capabilities. The dark blue boxes in the second and third rows present appropriate use of the capabilities and is a set of configuration that is managed by the institutional administrator. 